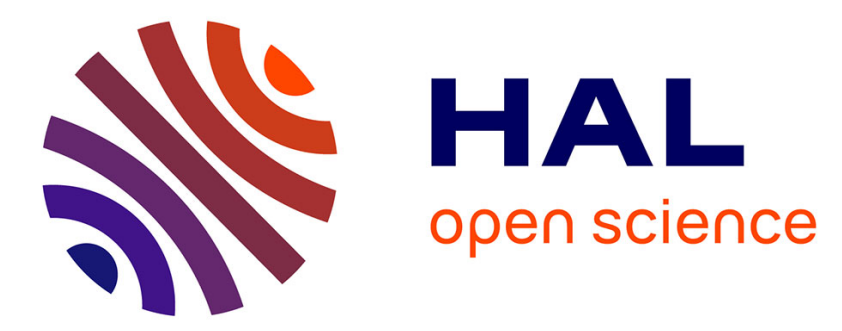

\title{
Super-resolved local recruitment of CLDN5 to filtration slits implicates a direct relationship with podocyte foot process effacement
}

\author{
Florian Tesch, Florian Siegerist, Eleonora Hay, Nadine Artelt, Christoph \\ Daniel, Kerstin Amann, Uwe Zimmermann, Panagiotis Kavvadas, Olaf Grisk, \\ Christos Chadjichristos, et al.
}

\section{To cite this version:}

Florian Tesch, Florian Siegerist, Eleonora Hay, Nadine Artelt, Christoph Daniel, et al.. Super-resolved local recruitment of CLDN5 to filtration slits implicates a direct relationship with podocyte foot process effacement. Journal of Cellular and Molecular Medicine, 2021, 10.1111/jcmm.16519 . hal03268855

\section{HAL Id: hal-03268855 \\ https://hal.sorbonne-universite.fr/hal-03268855}

Submitted on 23 Jun 2021

HAL is a multi-disciplinary open access archive for the deposit and dissemination of scientific research documents, whether they are published or not. The documents may come from teaching and research institutions in France or abroad, or from public or private research centers.
L'archive ouverte pluridisciplinaire HAL, est destinée au dépôt et à la diffusion de documents scientifiques de niveau recherche, publiés ou non, émanant des établissements d'enseignement et de recherche français ou étrangers, des laboratoires publics ou privés. 


\title{
Super-resolved local recruitment of CLDN5 to filtration slits implicates a direct relationship with podocyte foot process effacement
}

\author{
Florian Tesch $^{1}$ | Florian Siegerist ${ }^{1}$ (D) | Eleonora Hay ${ }^{1,2}$ | Nadine Artelt ${ }^{1}$ | \\ Christoph Daniel $^{3}$ | Kerstin Amann ${ }^{3}$ | Uwe Zimmermann ${ }^{4}$ | Panagiotis Kavvadas ${ }^{5}$ | \\ Olaf Grisk $^{6}$ | Christos Chadjichristos ${ }^{5}$ | Karlhans Endlich ${ }^{1}$ | Christos Chatziantoniou ${ }^{5}$ | \\ Nicole Endlich ${ }^{1}$
}

${ }^{1}$ Department of Anatomy and Cell Biology, University Medicine Greifswald, Greifswald, Germany

${ }^{2}$ Department of Mental and Physical Health and Preventive Medicine, Section of Human Anatomy, University of Campania "Luigi Vanvitelli", Naples, Italy

${ }^{3}$ Department of Nephropathology, Institute of Pathology, University of ErlangenNuremberg, Erlangen, Germany

${ }^{4}$ Department of Urology, University Medicine Greifswald, Greifswald, Germany

${ }^{5}$ UMRS 1155, INSERM, Sorbonne Université, Paris, France

${ }^{6}$ Institute for Physiology, Medizinische Hochschule Brandenburg Theodor Fontane, Neuruppin, Germany

\section{Correspondence}

Nicole Endlich, Department of Anatomy and Cell Biology, Friedrich-Loeffler-Str. 23c, Greifswald 17487, Germany.

Email: nicole.endlich@uni-greifswald.de

Funding information

Federal Ministry of Education and Research, Grant/Award Number: 01GM1518B; Südmeyer fund for kidney and vascular research; Dr Gerhard Büchtemann fund; Open Access funding enabled and organized by Projekt DEAL.WOA Institution: N/A; Blended DEAL: Projekt DEAL

\begin{abstract}
Under healthy conditions, foot processes of neighbouring podocytes are interdigitating and connected by an electron-dense slit diaphragm. Besides slit diaphragm proteins, typical adherens junction proteins are also found to be expressed at this cell-cell junction. It is therefore considered as a highly specialized type of adherens junction. During podocyte injury, podocyte foot processes lose their characteristic 3D structure and the filtration slits typical meandering structure gets linearized. It is still under debate how this change of structure leads to the phenomenon of proteinuria. Using super-resolution 3D-structured illumination microscopy, we observed a spatially restricted up-regulation of the tight junction protein claudin-5 (CLDN5) in areas where podocyte processes of patients suffering from minimal change disease (MCD), focal and segmental glomerulosclerosis (FSGS) as well as in murine nephrotoxic serum (NTS) nephritis and uninephrectomy DOCA-salt hypertension models, were locally injured. CLDN5/nephrin ratios in human glomerulopathies and NTStreated mice were significantly higher compared to controls. In patients, the CLDN5/ nephrin ratio is significantly correlated with the filtration slit density as a foot process effacement marker, confirming a direct association of local CLDN5 up-regulation in injured foot processes. Moreover, CLDN5 up-regulation was observed in some areas of high filtration slit density, suggesting that CLND5 up-regulation preceded the changes of foot processes. Therefore, CLDN5 could serve as a biomarker predicting early foot process effacement.
\end{abstract}

\section{KEYWORDS}

3D-structured illumination microscopy, Claudin-5, foot process effacement,

glomerulosclerosis, podocyte, tight junction proteins

This is an open access article under the terms of the Creative Commons Attribution License, which permits use, distribution and reproduction in any medium, provided the original work is properly cited.

(c) 2021 The Authors. Journal of Cellular and Molecular Medicine published by Foundation for Cellular and Molecular Medicine and John Wiley \& Sons Ltd. 


\section{1 | INTRODUCTION}

Interdigitating podocyte foot processes are an essential part of the kidney filtration barrier. The filtration slit (FS) between foot processes is bridged by the slit diaphragm (SD) which is structured by homodimerization of the transmembrane protein nephrin. ${ }^{1}$ At early time points during glomerular development, podocyte precursor cells are connected by tight junctions. ${ }^{2}$ Later, at the capillary loop stage, the number of occluding junctions reduces, and the SD is finally formed. Additionally, other filtration slit-associated proteins like NEPH $1,{ }^{3}$ podocin, ${ }^{4}$ CD2AP ${ }^{5}$ or mFAT1, ${ }^{6}$ which are essential for the size selectivity of the glomerular filtration barrier, are expressed in podocytes and localize to the SD.

This unique cell-cell contact was also referred by Farquahr et al as a specialized type of adherens junction ${ }^{7}$. Farquhar co-workers have further shown that after the onset of a glomerular disease, which is associated with foot processes effacement, the composition of this specific cell-cell contact changes and converts back to occluding junctions indicating a de-differentiation of this post-mitotic cell type. $^{2,7}$ These morphological changes of the foot processes could be studied in detail by electron microscopy in the past; however the exact molecular composition of these occluding junctions in interdigitating podocytes remained unclear for decades. In 2009 it was demonstrated that the tight junction proteins JAM-A, occludin and cingulin are expressed at the FS of healthy rat podocytes. ${ }^{8}$ Additionally, in puromycin aminonucleoside nephropathy (PAN) rats, a well-established model to induce podocyte foot process effacement, these tight junction proteins were significantly up-regulated. This suggested a basic mechanism of tight junction formation in nephrotic kidneys.

Besides these results, it was reported that claudin-5 (CLDN5), a tetraspanning transmembrane protein of the claudin family, ${ }^{9,10}$ is a major component of tight junction complexes. Koda et $\mathrm{al}^{11}$ who investigated CLDN5 in rats and mice have clearly shown by in situ hybridization that CLDN5 mRNA is expressed in podocytes, in endothelial cells of the arterioles at the glomerular vascular pole as well as in the endothelium of larger arteries. Transmission electron microscopy (TEM) images further showed that CLDN5 is localized at the SD of control rats as well as of rats with PAN-induced foot process effacement. Surprisingly, Western blot analysis of isolated glomeruli revealed that CLDN5 is not up-regulated after PAN treatment. This is contrastive to other SD-associated tight junction proteins like JAM-A, occludin and cingulin that were significantly regulated in glomerular disease. ${ }^{8}$

In this present study, we wanted to analyse whether we can resolve the localization of a podocyte-specific claudin on the level of individual foot processes. Furthermore, we wanted to prove our hypothesis that the de novo localization of the tight junction protein CLDN5 precedes structural changes of podocyte foot processes and therefore indicates dedifferentiation of podocytes.

Since healthy podocyte foot processes are at the borderline of resolvability of conventional (fluorescence) light microscopes, which is limited to $200 \mathrm{~nm}$ in the lateral direction, electron microscopy has been the gold standard for its visualization for decades. However, this technique is time-consuming, highly sophisticated and can only analyse a thin layer of 50-90 nm of a kidney section. To overcome this obstacle, our group has established the 3D analysis of podocyte FS by the super-resolution microscopy technique 3D-SIM with $4 \mu \mathrm{m}$ paraffin-embedded tissue sections obtained from the diagnostic routine. ${ }^{12}$

Herein, we revealed by 3D-SIM that the podocyte-specific CLDN5 has a specific staining pattern in healthy and diseased kidneys that can be resolved by 3D-SIM, Moreover, we suggest CLDN5 as a possible early effacement marker.

\section{2 | MATERIALS AND METHODS}

\section{1 | Kidney tissue}

For our study formalin-fixed paraffin-embedded (FFPE) human kidney biopsies of patients diagnosed with $\operatorname{MCD}(n=5)$ and FSGS ( $n=5$ ) by experienced pathologists of the Department of Nephropathology of University Erlangen-Nuremberg were used. As healthy controls, anonymised excess normal kidney tissue of partial nephrectomies of the Department of Urology of the University Medicine Greifswald was used $(n=4)$. The use of the biopsies from Erlangen has been approved by the Ethics Committee of the Friedrich Alexander University of ErlangenNuremberg, waiving the need for retrospective consent for the use of archived excess material (Ref. No. 4415). The use of this excess kidney tissue from Greifswald has been approved by the Ethics Committee of the University Medicine Greifswald (Ref. No. BB 075/14). All patients stated written informed consent. All experiments were performed in accordance with local guidelines overseen by the University Medicine Greifswald and University Greifswald, Greifswald, Mecklenburg-Western Pomerania. Four micrometre sections were cut and mounted on Superfrost slides (R. Langenbrinck $\mathrm{GmbH}$ ).

Ten-week-old male C57BL/6 mice were retro-orbitally injected with $17 \mu \mathrm{L}$ of nephrotoxic serum (NTS) per gram of bodyweight over two consecutive days ( $n=7$ ) or with PBS as control $(n=3)$. On day 12 , mice were anesthetized with ketamine ( $100 \mathrm{mg} / \mathrm{kg}$ bodyweight)xylazine (10 mg/kg bodyweight) and killed and kidneys were harvested, formalin-fixed and paraffin-embedded. $4 \mu \mathrm{m}$ sections were cut and mounted on \#1.5 High Precision coverslips (Paul Marienfeld $\mathrm{GmbH})$. All animal experiments were performed in strict accordance with good animal practice and animal research guidelines and were approved by the appropriate committee of the Institut national de la santé et de la recherche médicale (INSERM) and the Pierre and Sorbonne Université Paris, France (Ref. No. B752001). FFPE kidney tissue of the uninephrectomy DOCA salt hypertension mouse model of a previous study was used which was prepared as described before ${ }^{13}$ 


\section{2 | Immunofluorescence staining}

After deparaffinization in xylene and rehydration in a descending ethanol series, all sections were boiled with a pressure cooker in Tris EDTA buffer ( $10 \mathrm{mmol} / \mathrm{L}$ Tris, $1 \mathrm{mmol} / \mathrm{L}$ EDTA, bring to $\mathrm{pH}=9$ using $\mathrm{NaOH}$, add $500 \mu \mathrm{L}$ Tween 20) for antigen retrieval. After autofluorescence quenching in $100 \mathrm{mmol} / \mathrm{L}$ glycine diluted in purified water and three times washing in PBS, slices were incubated for 1 hour in blocking solution (1\% FBS, 1\% BSA, $0.1 \%$ fish gelatin, $1 \%$ normal goat serum in PBS). The primary antibodies (1:400 in blocking solution, polyclonal guinea pig anti-nephrin IgG GP-N2, Progen; 1:400 in blocking solution, monoclonal mouse anti-Claudin-5 IgG, 4C3C2, Thermo Fisher Scientific; 1:50 in blocking solution, polyclonal rabbit anti-CD31 lgG, ab28364, Abcam) were incubated at $4^{\circ} \mathrm{C}$ on the slides overnight. The next day after five times washing in PBS and blocking for 45 minutes, the secondary antibodies (all 1:600 in blocking solution: Cy3-conjugated donkey anti-guinea pig IgG $(\mathrm{H}+\mathrm{L})$, 706-165-148, Jackson Immuno Research, Hamburg, Germany; Alexa Fluor 488-conjugated $\mathrm{F}(\mathrm{ab})_{2}$-fragment goat anti-mouse IgG, 115 546-072, Jackson Immuno Research; Alexa Fluor 647-conjugated $\mathrm{F}(\mathrm{ab})_{2}$-fragment goat anti-rabbit IgG, 111-607-047, Jackson Immuno Research, Hamburg, Germany) were incubated at $4^{\circ} \mathrm{C}$ for 45 minutes, followed by five times washing in PBS and transfer to purified water. The slices were mounted in Mowiol for Microscopy (Carl Roth) using High Precision cover glasses (Paul Marienfeld GmbH).

\section{3 | Microscopy}

For confocal laser scanning microscopy, a Leica TCS SP5 (Leica Microsystems) equipped with a $63 \times(N A 1.4)$ oil immersion objective was used. Mouse glomeruli were imaged with 6.080 pixel/ $\mu \mathrm{m}$. Human glomeruli were imaged with $5.853 \mathrm{pixel} / \mu \mathrm{m}$. For 3DSIM a Zeiss Elyra PS.1 System (Zeiss Microscopy) equipped with a $63 \times$ (NA 1.4) oil immersion objective was used. Z-Stacks with a size of $1280 \mu \mathrm{m} \times 1280 \mu \mathrm{m}$ with a slice-to-slice distance of $0.2 \mu \mathrm{m}$ were acquired over approximately $4 \mu \mathrm{m}$ using the $561 \mathrm{~nm}$ laser, (3\% laser power, exposure time:100 ms) and the $488 \mathrm{~nm}$ laser (4\% laser power, exposure time: $100 \mathrm{~ms}$ ). The $28 \mu \mathrm{m}$ period grating was shifted five times and rotated five times on every frame while acquiring widefield images. The 3D-SIM reconstruction was performed with the Zeiss ZEN Black Software using following parameters: Baseline Cut, SR Frequency Weighting: 1.0; Noise Filter: -5.6; Sectioning: 96, 81, 81. ZEN Blue software was used for maximum intensity projections.

\section{4 | CLDN5/nephrin ratio measurement}

Z-stacks of glomeruli stained for nephrin and CLDN5 were transformed into maximum intensity projections. For automated measurement of the CLDN5/nephrin ratio, a customized macro for the ImageJ-based platform FIJI was programmed. A FS area is selected manually. The macro performs binarization of the nephrin-Cy3- and the CLDN5-Alexa488-channel according to defined thresholds creating black and white images. The nephrin- and CLDN5-positive area is measured and saved as an Excel file. With these data, the CLDN5/ nephrin ratio of the area was calculated. The CLDN5/nephrin ratio was evaluated for a minimum of 20 areas per glomerulus, and the mean was calculated. To check for statistical difference between effaced kidney samples and controls, not completely normally distributed values were compared using Mann-Whitney $U$ test using SPSS Statistics (25.0 IBM SPSS Inc).

\subsection{Correlation of CLDN5/nephrin ratio with filtration slit density}

Filtration slit density (FSD) was measured with Podocyte Effacement Measurement Procedure (PEMP) as published before. ${ }^{12}$ Correlation analysis between CLDN5/nephrin ratio and FSD was performed using Spearman's correlation for not normally distributed data using SPSS Statistics. All graphs were set up with Prism 5.01 (GraphPad).

\section{3 | RESULTS}

\section{1 $\mid$ CLDN5 is expressed in podocyte foot processes}

To investigate which claudins are expressed in a podocyte-specific manner, we performed a database analysis using the Kidney Cell Explorer (https://cello.shinyapps.io/kidneycellexplorer/) created by Ransick et al $^{14}$, which is based on a single-cell RNA sequencing data set of murine kidneys. Of all claudins annotated in the database, we found that Claudin-5 (CLDN5) transcripts specifically clustered in the podocyte population whereas the other claudins were expressed more distally in the nephron (Figure 1A, Figure S1). We verified this finding in a microarray dataset (http://www.nephroseq.org, 02/2020, University of Michigan, Ann Arbor, MI) of human isolated glomeruli (Figure 1B), in which CLDN5 was statistically significant enriched in glomeruli in comparison to the tubular kidney fraction. ${ }^{15}$

Furthermore, the Proteinatlas database ${ }^{16}$ (http://www.proteinatl as.org) was used and the expression of the five most proximal expressed claudins CLDN1, 2, 5, 11, 12 was evaluated by immunohistochemistry (IHC) of kidney tissue (Figure 1C). As described before, we found that CLDN1 was expressed within parietal epithelial cells in contrast to CLDN5 which is expressed by cells on the glomerular tuft. As shown in Figure $1 \mathrm{C}$, the IHC using polyclonal anti-CLDN5 antibodies showed positivity in glomerular cells, with a pronounced linear staining pattern along the glomerular capillaries indicating a localization of CLDN5 in either podocyte foot processes and/or endothelial cells together with weaker and speckled staining in the tubulointerstitium. The other claudins were expressed mainly nonglomerular and in the tubular system, respectively.

To further localize CLDN5 within the healthy kidney, we stained kidney sections of human nephrectomy samples and healthy 

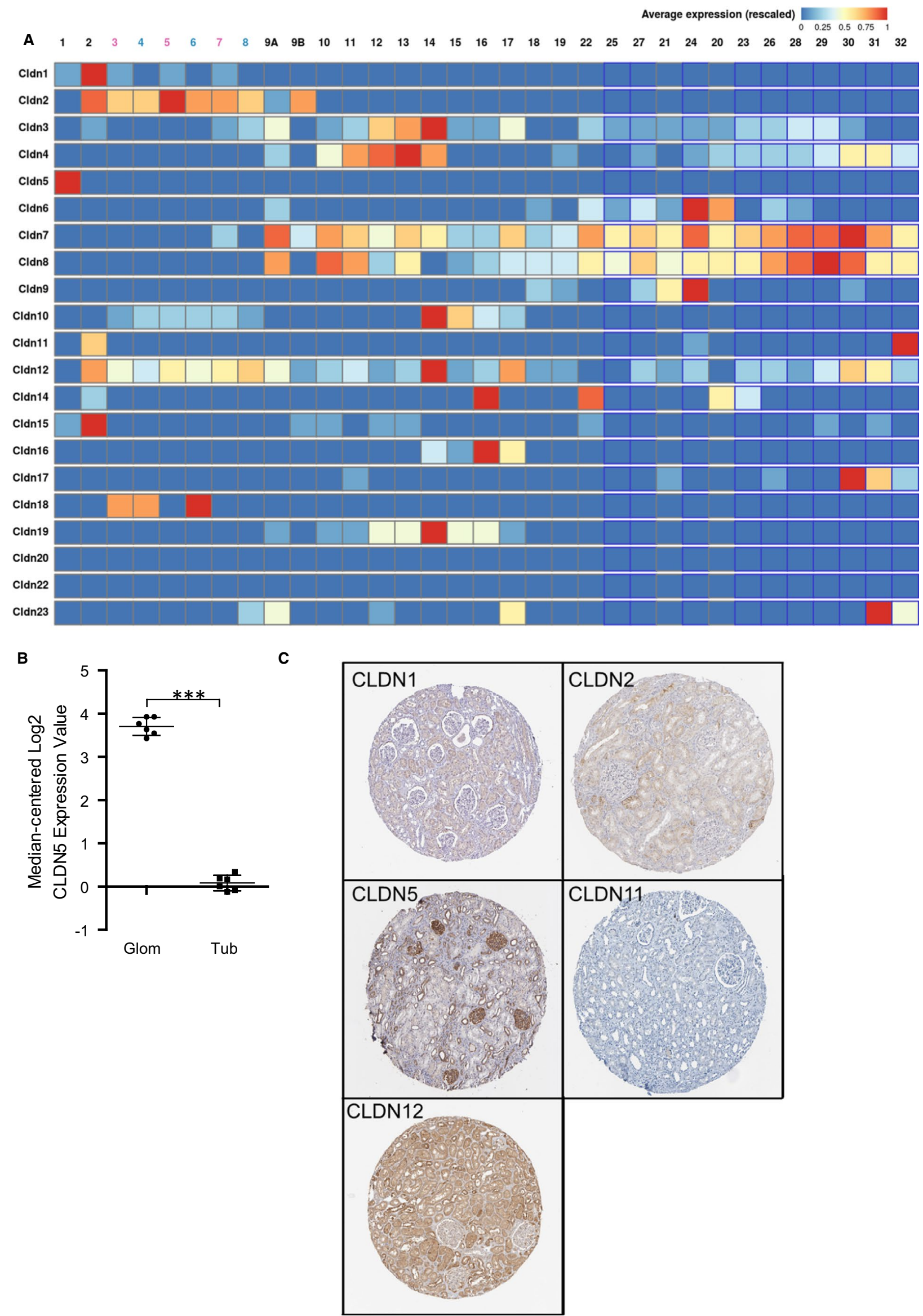
FIGURE 1 A, Database analysis of CLDN1 to CLDN23 using the Kidney Cell Explorer by Ransick et al ${ }^{14}$ based on a single-cell RNA sequencing data set of murine kidneys to determine podocyte-specific claudins. ID 1 represents podocytes, ID 2 represents parietal epithelial cells and ID 3 following represents tubular cells, as explained in Figure S1 CLDN5 is strongly expressed in ID 1 of the nephron representing podocytes. Other claudins were expressed further distally in the nephron or not expressed at all. B, The results were verified by analysis of the CLDN5 expression based on isolated glomeruli by Lindenmeyer et al, ${ }^{15}$ which showed a significantly higher mediancentered Log2 CLDN5 expression in the glomerulus compared to the tubule apparatus. ${ }^{* * *} P<.001 \mathrm{C}$, Evaluation of immunohistochemistry stainings of CLDN1, 2, 5, 11 and 12 from the Proteinatlas database ${ }^{16}$ to investigate murine glomerular claudin expression. CLDN1 was expressed in parietal epithelial cells but not in podocytes. CLDN5 was expressed broadly in the glomerulus with accentuated linear staining along the glomerular capillaries indicating localization to podocyte foot processes or the slit diaphragm. CLDN 2 and 12 were expressed mainly in the tubular system. CLDN 11 showed overall low antibody enhancement in kidney sections

FIGURE 2 C-LSM images of healthy human glomeruli showed CLDN5 expression in the FS and the endothelium of glomerular capillaries (arrows) and of arterioles at the vascular pole (arrowheads). In mouse glomeruli, CLDN5 was expressed in the FS and at the vascular pole (arrowheads), but no expression was found in glomerular capillaries. The scale bars indicate $20 \mu \mathrm{m}$

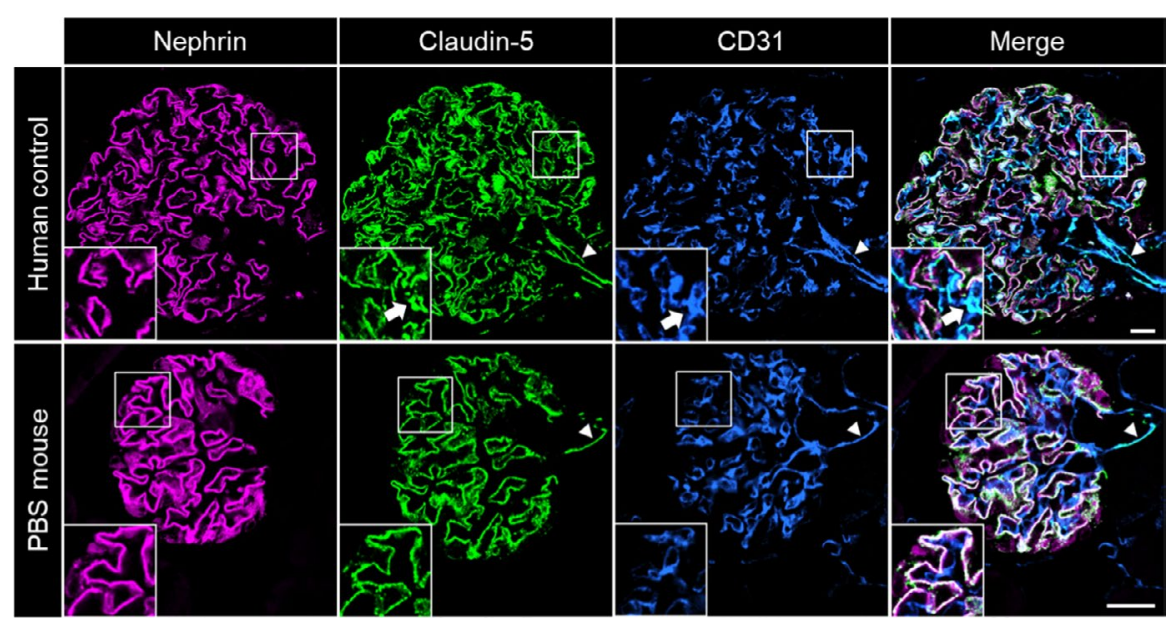

wild-type mice with a monoclonal antibody directed against CLDN5 and detected this antibody with fluorescence-labelled secondary antibodies. In confocal laser scanning micrographs (C-LSM) of healthy human samples, we found CLDN5 localized at the podocyte slit membrane partially colocalized with the slit diaphragm protein nephrin, as well as the glomerular endothelium identified by a co-staining against CD31. Additionally, we found CLDN5 localized in the arterioles of the glomerular vascular pole. In sections of control mice, the CLDN5 expression was also found at the $\mathrm{SD}$ and vascular pole, but no expression was found in glomerular capillaries (Figure 2).

\section{2 | 3D-SIM studies of CLDN5}

To further determine the subcellular localization of CLDN5 beyond the diffraction limit in podocytes, we used 3D-SIM of formalin-fixed paraffin-embedded (FFPE) tissue samples which allows high resolution fluorescence imaging of podocyte foot processes. With our experimental set-up and sample preparation, we reached an $x y$ resolution down to $115 \mathrm{~nm}$, which is sufficient to resolve individual healthy podocyte foot processes and the slit diaphragm (Figure S2). In 3D-SIM images of healthy human and mouse samples CLDN5 focally co-localized with the slit diaphragm protein nephrin along the FS (Figure 3, Figure 4).

\subsection{CLDN5 is up-regulated in MCD and FSGS}

To find out whether CLDN5 was regulated in glomerular disease, we screened disease-specific datasets within the aforementioned Nephroseq database. We found CLDN5 to be statistically significant up-regulated 2.05-fold $(P=.005)$ in the Nakagawa CKD microarray cohort ${ }^{17}$ compared with healthy donor kidneys (Figure S3).

Then, we co-immunostained biopsy specimen of MCD and FSGS patients for CLDN5 and nephrin. Patient characteristics at time of biopsy together with serum creatinine and proteinuria are summarized in Table 1.

In contrast to healthy samples, patient biopsies showed extended CLDN5-positive lines that were localized in areas of effaced podocyte foot processes. Figure 3 shows more linearized and less meandering nephrin-positive filtrations slits, which can be used as a direct marker for broadened foot processes.

\subsection{CLDN5 and nephrin are inversely expressed in murine models of glomerular disease}

To investigate whether this up-regulation of CLDN5 is a speciesindependent and general mechanism rather than a human diseasespecific phenomenon, we imaged FFPE sections of mice (10 weeks old) with induced nephrotoxic serum (NTS) nephritis 12 days after 


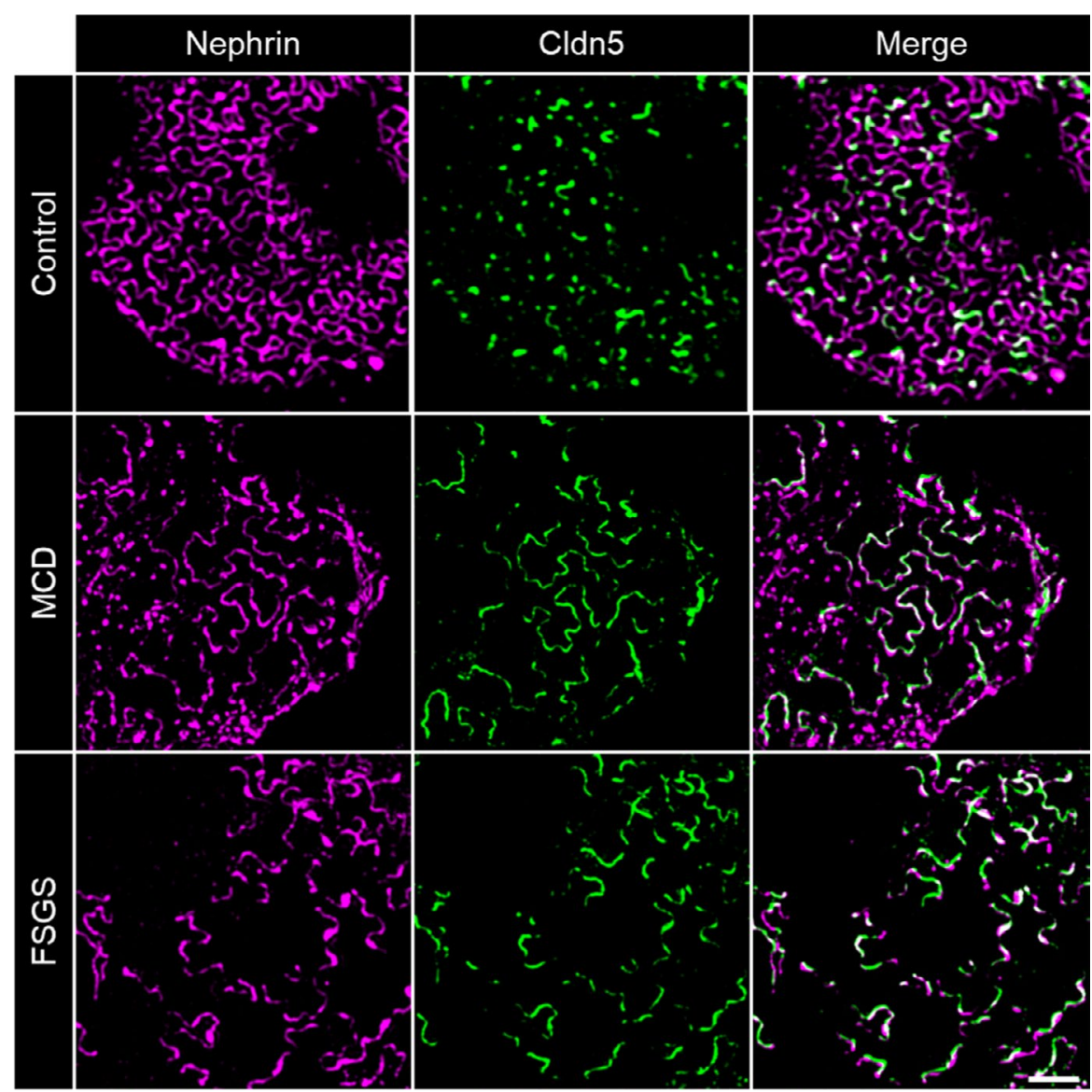

FIGURE 3 3D-SIM images of human glomeruli. CLDN5 focally co-localized with the slit diaphragm protein nephrin along the FS in human nephrectomy samples. MCD and FSGS glomeruli showed extended CLDN5-positive lines at the FS in areas of effaced podocyte foot processes. The scale bar indicates $2 \mu \mathrm{m}$
TABLE 1 Patient characteristics of the biopsies used in this study

\begin{tabular}{|lllll|}
\hline ID & $\begin{array}{l}\text { Age } \\
\text { (y) }\end{array}$ & $\begin{array}{l}\text { Sex } \\
(\mathrm{f} / \mathrm{m})\end{array}$ & Proteinuria (g/24 h) & $\begin{array}{l}\text { Serum } \\
\text { creatinine } \\
\text { (mg/dL) }\end{array}$ \\
\hline MCD 1 & 32 & $\mathrm{f}$ & 7 & 1.6 \\
\hline MCD 2 & 26 & $\mathrm{f}$ & 4 & $<1$ \\
\hline MCD 3 & 46 & $\mathrm{~m}$ & 1.4 & 2 \\
\hline MCD 4 & 36 & $\mathrm{~m}$ & 3.2 & 1.5 \\
\hline MCD 5 & 67 & $\mathrm{f}$ & 10 & 3 \\
\hline FSGS 1 & 73 & $\mathrm{~m}$ & 0.7 (g/g creatinine) & 1.67 \\
\hline FSGS 2 & 64 & $\mathrm{f}$ & 9 & 2.64 \\
\hline FSGS 3 & 71 & $\mathrm{~m}$ & 4 & 1.5 \\
\hline FSGS 4 & 37 & $\mathrm{~m}$ & 5 & 1.5 \\
\hline FSGS 5 & 80 & $\mathrm{f}$ & 13.8 & 2 \\
\hline
\end{tabular}

NTS-injection and corresponding control-treated mice. It is wellestablished that after NTS-injection, mice develop severe glomerulonephritis with heavy proteinuria accompanied by severe podocyte foot process effacement. Similar to the human biopsies, a massive local up-regulation of CLDN5 was found in the cell-cell junctions of effaced foot processes in NTS-injected mice (Figure 4A).
Furthermore, to investigate if the up-regulation of CLDN5 together with increasing foot process effacement, is a general rather than a model-specific phenomenon, we additionally evaluated sections of the uninephrectomy deoxycorticosterone acetate (DOCA)-salt hypertension mouse model. In this model, mice develop hypertension after uninephrectomy, parenteral DOCA application and enteral salt application. During the course of disease, mice develop progressive albuminuria and glomerulosclerosis similar to human secondary FSGS. As indicated in Figure 4B, we found that also in this model, CLDN5 was recruited to effaced FS. The local phenotype in effaced areas was similar to the previous findings whereas in general the phenotype in the DOCA-salt hypertension model was less uniform and more focally restricted to single glomeruli and not as distributed as in the NTS model.

Independent of the model or disease investigated, we observed that during the beginning of foot process effacement (phase 1), nephrin is still continuously localized at the FS followed by an up-regulation of CLDN5. Interestingly, effacement without CLDN5 up-regulation could only be found very rarely in some FS areas. With progressing effacement (phase 2), nephrin staining appeared discontinuous and CLDN5-positive but nephrin-negative FS areas were identified, especially in FSGS biopsies (Figure 5). The same was seen in NTS-treated mice (Figure S4) indicating the severity of the NTS-model with full dedifferentiation of the FS to a tight junction phenotype. 
FIGURE 4 3D-SIM images of mouse glomeruli. A, CLDN5 focally co-localized with nephrin along the FS in PBStreated control mice. Similar to human biopsies, glomeruli of mice treated with nephrotoxic serum (NTS) showed extended CLDN5-positive lines at the FS in areas of effaced podocyte foot processes. Panel B, shows focal CLDN5 localization to effaced FS in mice of the uninephrectomy deoxycorticosterone acetate (DOCA) salt hypertension mouse model compared with UNX controls. The scale bar indicates $2 \mu \mathrm{m}$
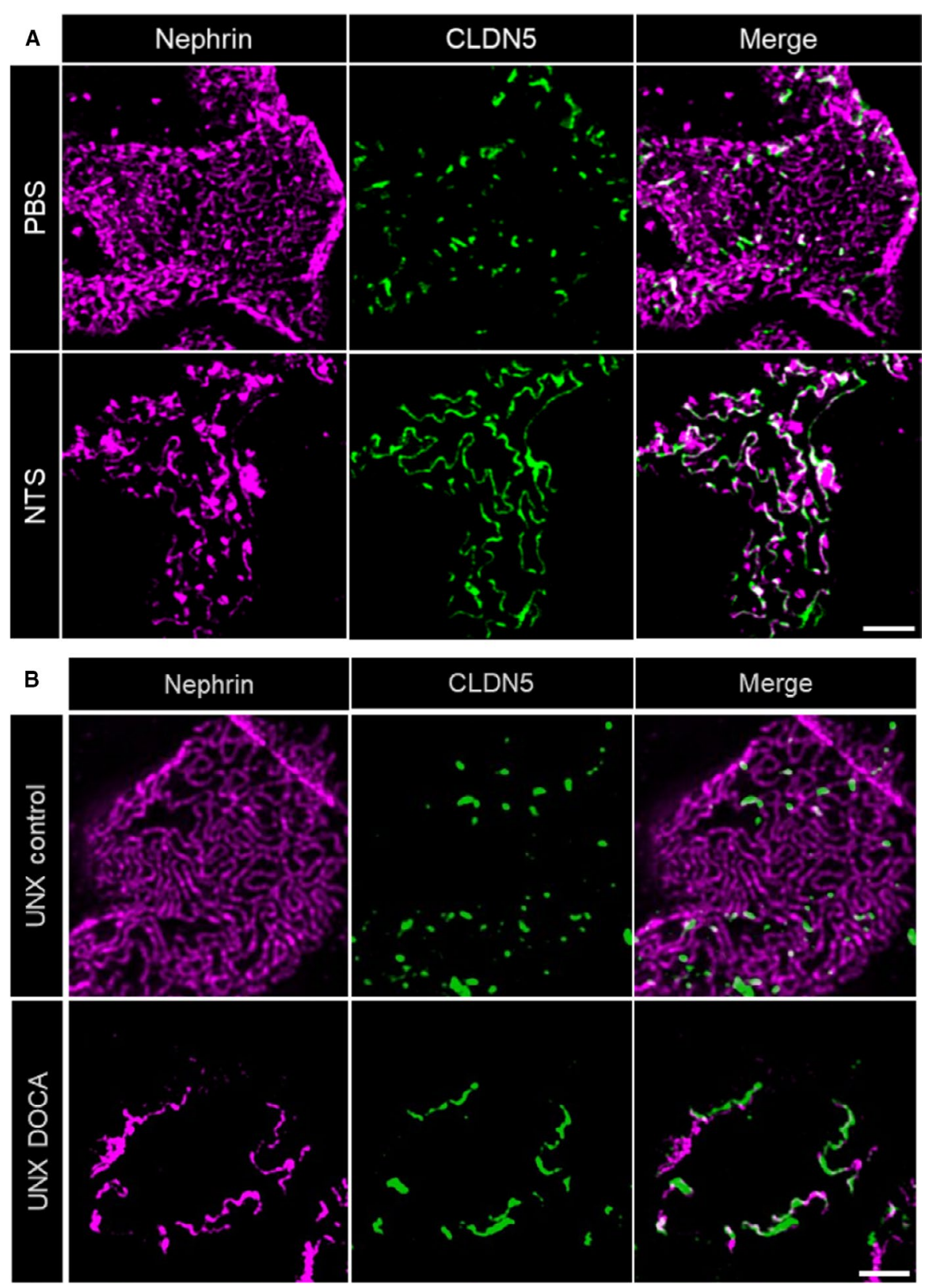

\subsection{CLDN5/nephrin ratio correlates with the FSD}

These results raised the question, whether a CLDN5 up-regulation could only be observed in strongly effaced FS areas or if it could be also observed in areas with a high filtration slit density (FSD). Although most healthy FS areas only showed focally localized CLDN5 expression, as described above, several FS areas with a high FSD (indicating healthy foot processes) seem to up-regulate CLDN5 (Figure 6).

To quantify these impressions, we developed a $\mathrm{FIJI}^{18}$-based macro to determine the CDLN5/nephrin ratio (Figure S5). Z-stacks were processed into maximum intensity projections. After selection of a plan FS area, thresholding and binarization of the CLDN5- and nephrin-channel, we quantified the CLDN5- and nephrin-positive areas. This allowed calculation of the CLDN5/nephrin ratio. As expected, we found a significantly higher CLDN5/nephrin ratio in MCD and FSGS samples than in human controls (Figure 7A) with no statistically significant difference between the MCD and FSGS biopsies investigated (Figure S6). These findings could be verified in mice as well: the CLDN5/nephrin ratio in NTS-injected mice was statistically significantly higher compared with PBS-injected control mice (Figure 7B).

The results showed a species-independent association of podocyte foot process effacement and CLDN5 up-regulation. To further investigate this association in detail, we measured the FSD with our established Podocyte Exact Morphology Measurement Procedure (PEMP). ${ }^{12}$ PEMP allows a quick and exact quantification of the FSD of each glomerulus where the CLDN5/nephrin ratio as determined before. Thereby, PEMP quantified the total length of the nephrin-positive FS $\left(I_{F S}\right)$ in a selected FS area as well as the size of the area (A). The FSD was calculated by dividing $I_{F S}$ by $A$ (Figure 7C). 


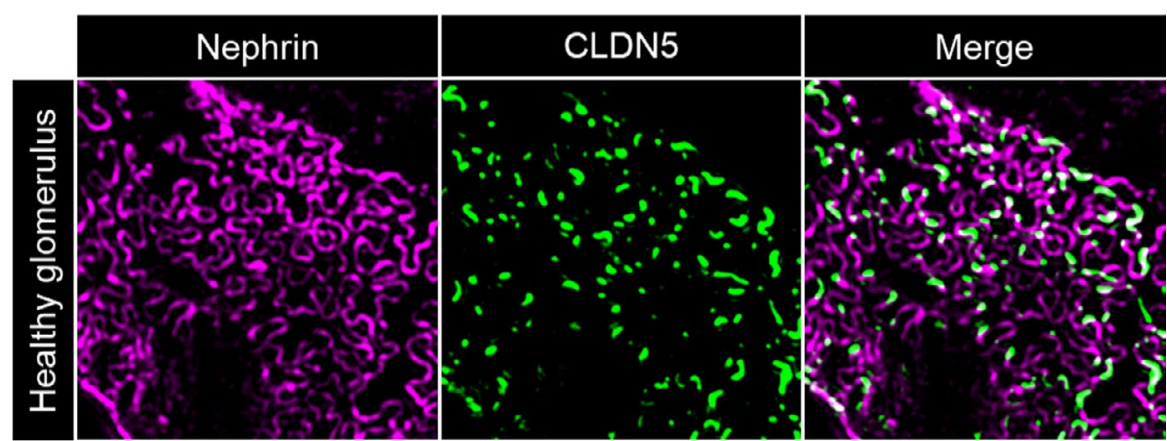

FIGURE 5 Evolution of CLDN5 upregulation in 3D-SIM images of human glomeruli. Compared with healthy glomeruli, in phase 1 of effacement CLDN5 was up-regulated following the nephrin-positive FS. Nephrin is continuous and effaced areas without CLDN5 could be observed (arrowheads). With progressing effacement (phase 2), nephrin became discontinuous and CLDN5-positive, but nephrin-negative areas were found (arrows), here shown in FSGS biopsies. The scale bar indicates $2 \mu \mathrm{m}$
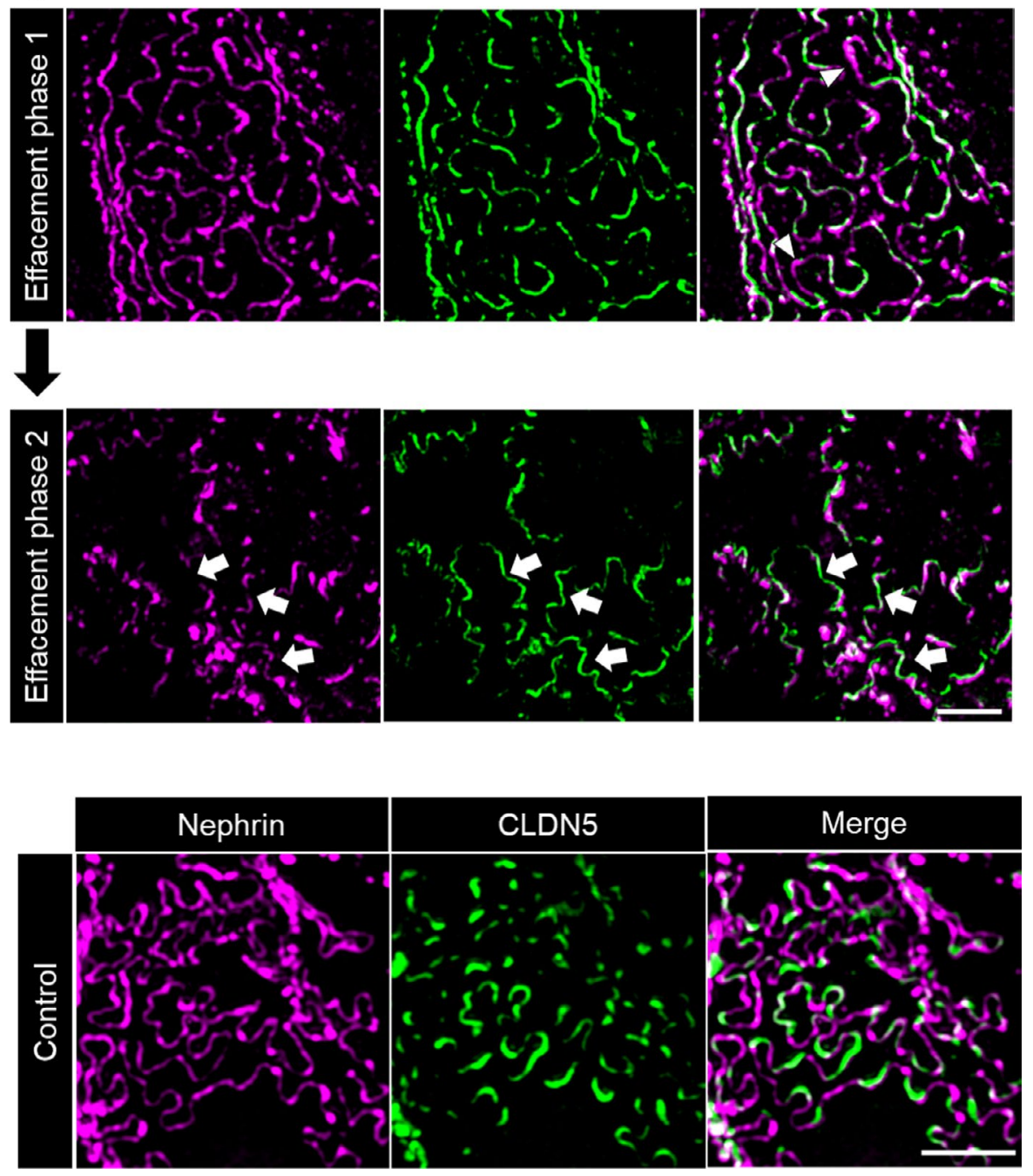

FIGURE 6 CLDN5 up-regulation in non-effaced FS areas of human control glomeruli. In several FS areas, CLDN5 forms continuous lines despite lack of foot process effacement and normal FSD. The scale bar indicates $2 \mu \mathrm{m}$

FIGURE 7 A, Results of CLDN5/nephrin ratio measurement showed a significantly higher CLDN5/nephrin ratio in MCD ( $\mathrm{n}=5$ ) and FSGS $(n=5)$ biopsies compared with human nephrectomy samples as controls $(n=4)$. Panel $B$, shows a significantly higher $C L D N 5 / n e p h r i n$ ratio in NTS-injected mice $(n=7)$ compared with PBS-injected control mice $(n=3)$. In A and $B$, every measuring point represents the glomerular mean of at least 20 measured areas. Error bars show standard deviation. ${ }^{* * * *} P<.0001,{ }^{* * *} P<.001,{ }^{* *} P<.01$, Mann-Whitney $U$ test. C, Measurement of the filtration slit density (FSD) in MCD $(n=5)$ and FSGS $(n=5)$ compared with human nephrectomy samples as controls $(n=4)$ with PEMP. D, MCD and FSGS biopsies showed a significantly reduced FSD compared with controls. Every measuring point represents the glomerular mean of at least 20 measured areas. Error bars show standard deviation. ${ }^{* * * *} P<.0001,{ }^{* * *} P<.001,{ }^{* *} P<.01$, Mann-Whitney $U$ test. E, Correlation analysis of CLDN5/nephrin ratio and FSD in MCD $(n=5)$, FSGS $(n=5)$ and controls $(n=4)$ showed a highly significant correlation of CLDN5/nephrin ratio and FSD. CLDN5 expression increased with increasing foot process effacement. Every measuring point represents the average for the whole sample. $r=-.952, P<.0001, R^{2}=.906$, Spearman's correlation 

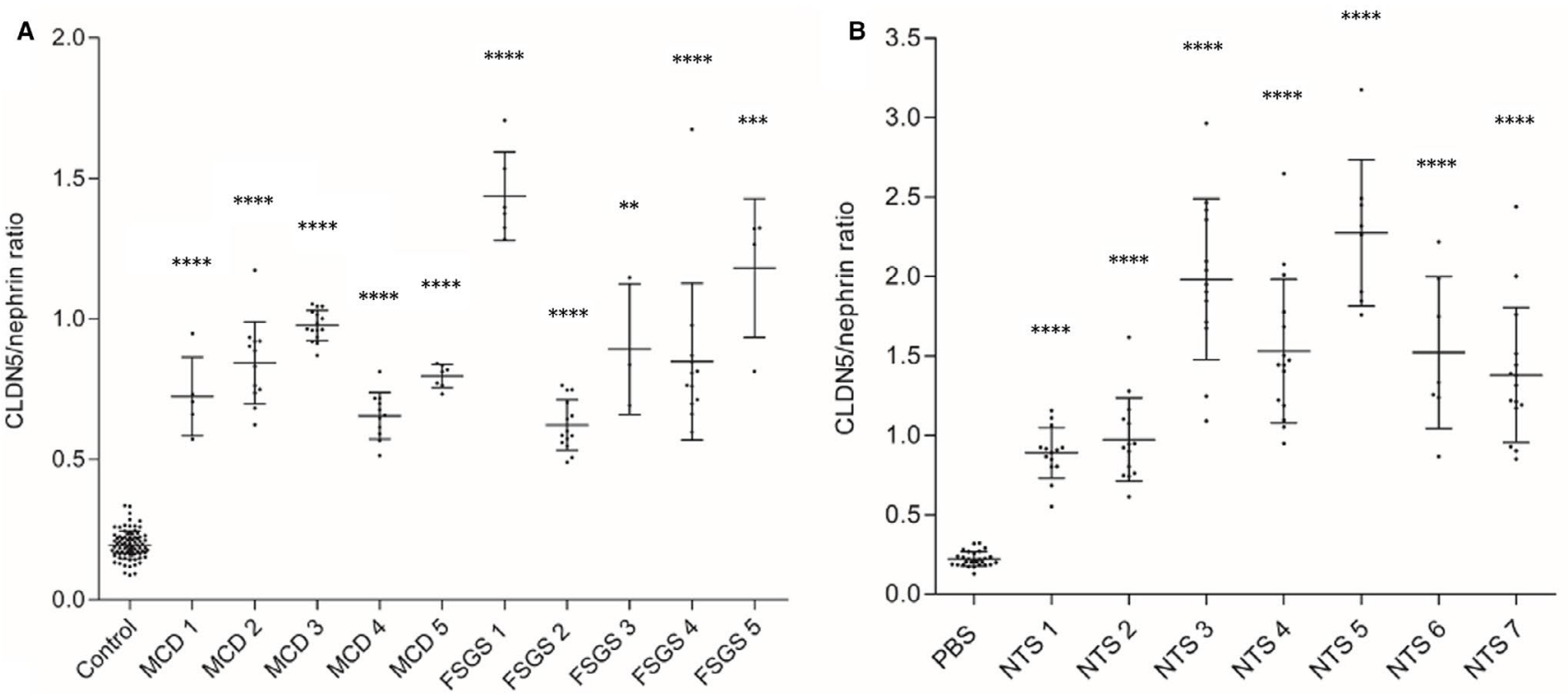

C
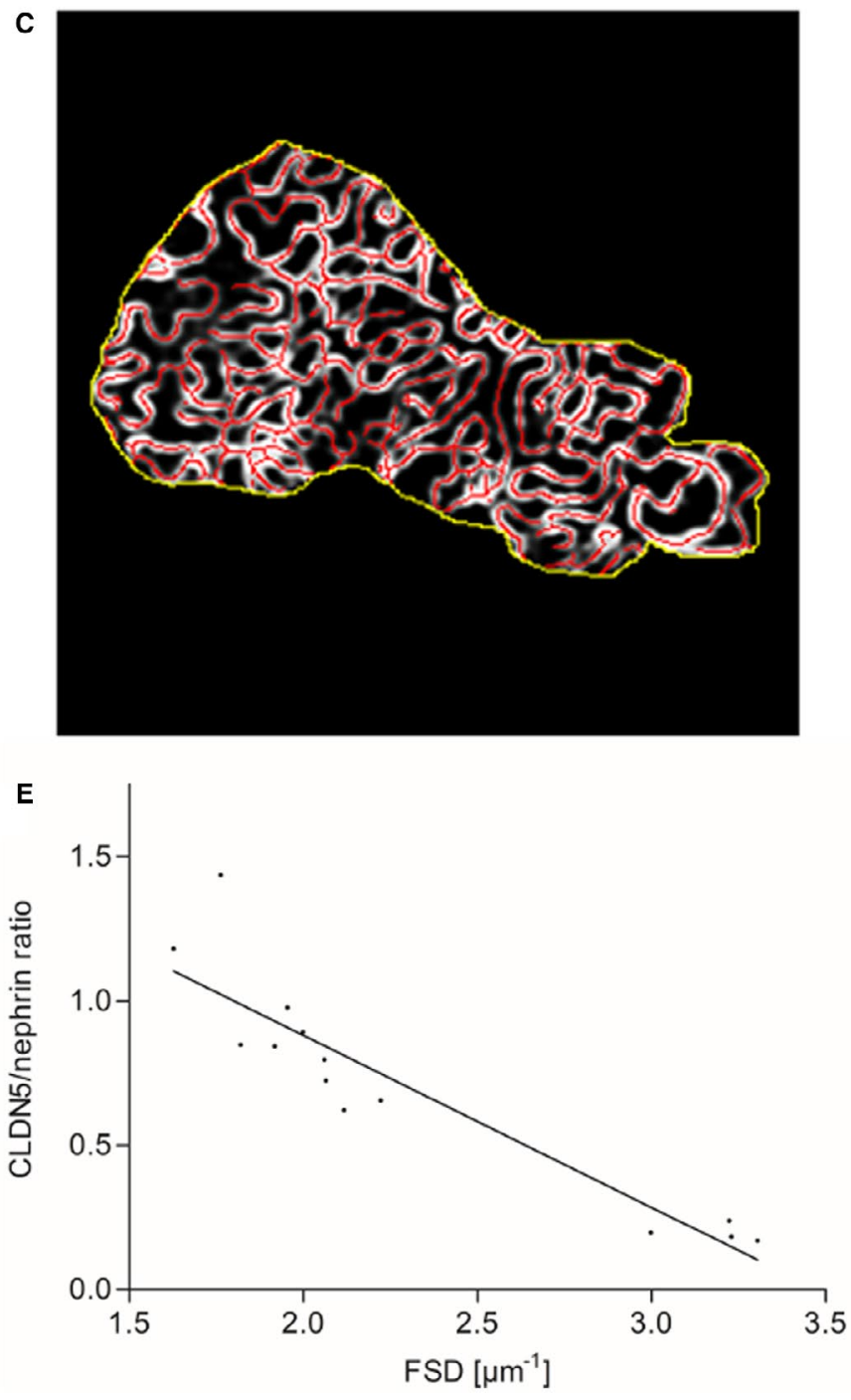

By using PEMP, we found that the FSD in MCD and FSGS samples was statistically significantly reduced compared with healthy human nephrectomy samples (Figure 7D). As shown in Figure 7E, CLDN5/
D

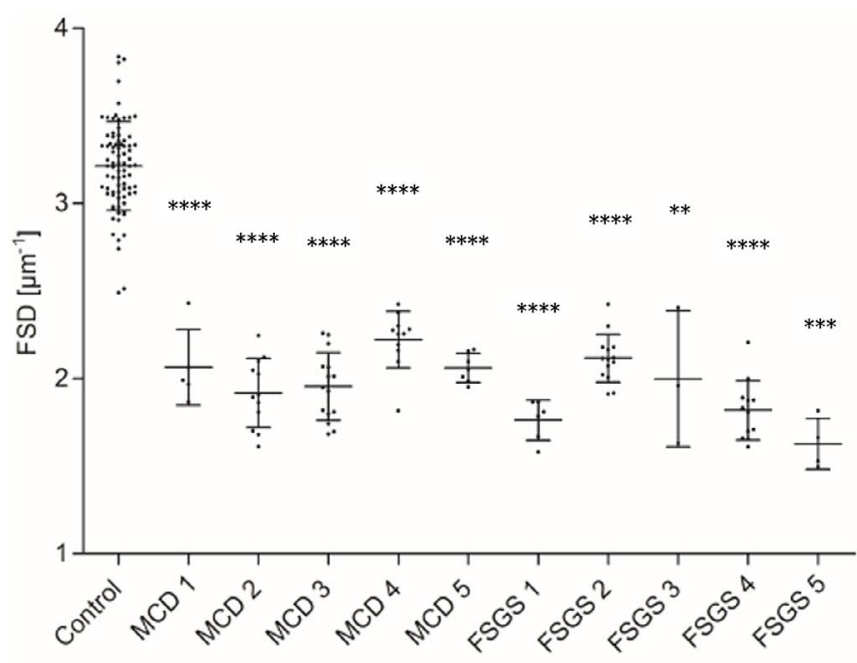

nephrin ratio and FSD correlated significantly $(r=-.952, P<.0001$, $R^{2}=.906$ ) in human samples, indicating that the CLDN5 localization in relation to the FS increased the broader the foot processes became. 


\section{4 | DISCUSSION}

To summarize, the super-resolving 3D-SIM technique revealed that CLDN5 was localized to those areas of the FS where foot processes were effaced. The CLDN5/nephrin ratio was significantly correlated with the FSD in human sections. We performed a super-resolution microscopy study of CLDN5 expression in human and mouse for the first time.

In our findings in mice, CLDN5 was not expressed in the capillary endothelium, but in the endothelia of arterioles of the vascular pole which was previously observed in rats as well. ${ }^{11}$ We found CLDN5 up-regulation in NTS mice compared with controls whereas Western blot analysis of CLDN5 expression in PAN-treated rats showed no up-regulation compared to controls. ${ }^{11}$ This may be traced back to the strong CLDN5 expression in arterioles of the vascular pole. Slight CLDN5 up-regulation in the FS of PAN-treated rats could not be detected by Western blot most probably because of superimposing CLDN5 expression in arterioles of isolated glomeruli.

This superimposing effect could be even stronger in human sections because of the additional CLDN5 expression in glomerular endothelial cells. In this context, one major advantage of our 3DSIM approach combined with FIJI-based evaluation is that CLDN5up-regulation in the FS can be studied separately from endothelial CLDN5 expression.

Furthermore, we confirmed the basic mechanism of tight junction formation in the FS in kidney injury for CLDN5. Tight junction protein up-regulation in the FS has been shown in PAN-treated rats before for occludin, cingulin and JAM-A. ${ }^{8}$ Lately, 3D-reconstruction of FIB/SEM images showed a co-localization of slit diaphragms and tight junctions in rats on day 4 after PAN treatment as well ${ }^{19}$ : as initial alteration slit diaphragms moved further to the luminal side of the FS while tight junctions formed below. In this stage, SDs and tight junctions coexisted in close proximity. On day 8 after PAN treatment, slit diaphragms were replaced by tight junctions which connected strongly effaced foot processes and slit diaphragms vanished. This could be an explanation for our finding that CLDN5 and nephrin were co-expressed and that the podocyte cell-cell contact shows tight junction formation with an up-regulation of CLDN5 whereas the nephrin-containing slit diaphragm still exists more luminal.

In our study of MCD and FSGS biopsies, continuous nephrin lines as well as a nephrin-CLDN5 co-localization could be seen despite effacement. It is conceivable that kidney injury in MCD and FSGS was not as strong as injury in rats following PAN treatment. In mice, NTS injection led to severe foot process effacement and fragmented nephrin lines whereas CLDN5 was continuous in these areas. For this reason, in several NTS mice CLDN5/nephrin ratios distinctly greater than 1 were measured indicating destruction of slit diaphragm and locally complete tight-junction transformation. By investigating CLDN5 expression in DOCA-treated unilateral nephrectomy mice, we could confirm CLDN5 recruitment to effaced FS areas as a general mechanism of murine kidney injury, rather than an NTS-specific phenomenon. Taken together, CLDN5 up-regulation was observed species- and model-independently in effaced FS areas.
In addition, our data showed FS areas of high FSD, where upregulation of CLDN5 was observed. Importantly, in these areas CLDN5 up-regulation was found without significant foot process effacement. Although in general CLDN5 expression increased with increasing effacement, these findings show that CLDN5 formation at the FS can precede the morphologic process of foot process effacement. For this reason, CLDN5 could be described as a potential early effacement marker predicting changes in foot process morphology. These results could only be discovered in control sections, because the MCD and FSGS samples investigated were already globally effaced. These results are tempting to hypothesize that tight junction formation and CLDN5 localization to the filtration slit precedes podocyte effacement and deposition of extracellular matrix forming sclerotic lesions. If this would be the case, this finding would be helpful to predict reoccurrence of glomerulosclerosis in kidney allografts. As in a usual clinical setting in naïve kidney biopsies rather show end-points of a preceding disease course, a more longitudinal study design, for example with protocol biopsies after kidney transplantation has to be used. Therefore, this conclusion could not be drawn in this biopsy set but will be under further investigation in a larger cohort.

As demonstrated by the proposed different phases of foot process effacement, CLDN5 and nephrin co-localized in healthy glomeruli and in early effaced foot processes. Therefore, it is conceivable that in those spots, CLDN5 is located in a non-TJ cell-cell contact. Earlier, Koda et $\mathrm{al}^{11}$ found CLDN5 ultrastructurally localized to intact filtration slits. It is tempting to speculate that the formation of foot process effacement and conversion into a TJ begins in spots in which CLDN5 focally localizes to the FS. As explained above, Ichimura and colleagues demonstrated a distinct vertical distance between simultaneously occurring slit diaphragms and tight junctions in the FS. To eliminate the influence of CLDN5 and nephrin being localized in slightly different planes of the z-stack, maximum intensity projections were used. Manual creation of maximum intensity projections guarantees that no CLDN5-signal from capillary endothelium is projected into the FS area, which otherwise would lead to falsely high CLDN5/nephrin ratios. To avoid selection bias, CLDN5/nephrin ratio and FSD were measured in all evaluable FS areas. Only glomeruli with a minimum of 20 evaluable FS areas were included in this study.

In conclusion, our study showed CLDN5 localization to effaced areas of the FS in human and mouse glomerulopathies. Highly significant correlation between FSD and CLDN5/nephrin ratio in human sections could be demonstrated. Our results show that with increasing effacement CLDN5 expression in the FS is increased simultaneously, whereas in several FS areas CLDN5 up-regulation preceded foot process effacement. CLDN5 can therefore be described as a marker predicting early foot process effacement. Our results hereby provide a better understanding of the sequence of tight junction formation in kidney injury.

\section{ACKNOWLEDGEMENTS}

This work was supported by a scholarship from the Gerhard Domagk program of the University Medicine Greifswald to FT and by a grant of the Federal Ministry of Education and Research (BMBF, grant 
01GM1518B, STOP-FSGS) to NE This work was generously supported by the Südmeyer fund for kidney and vascular research ('Südmeyer-Stiftung für Nieren- und Gefäßforschung') and the $\mathrm{Dr}$ Gerhard Büchtemann fund, Hamburg, Germany. The technical assistance of Mandy Weise is highly acknowledged.

\section{CONFLICTS OF INTEREST}

NE serves as CEO and holds shares, and FS holds shares of NIPOKA $\mathrm{GmbH}$, a company commercializing the PEMP approach. All other authors declare no conflicts of interest.

\section{AUTHOR CONTRIBUTIONS}

Florian Tesch: Formal analysis (equal); Funding acquisition (equal); Investigation (lead); Visualization (equal); Writing-original draft (equal); Writing-review and editing (equal). Florian Siegerist: Conceptualization (equal); Formal analysis (equal); Investigation (equal); Methodology (lead); Software (equal); Visualization (equal); Writing-original draft (equal); Writing-review and editing (equal). Eleonora Hay: Investigation (equal); Visualization (equal); Writingreview and editing (equal). Nadine Artelt: Investigation (equal); Methodology (equal); Resources (equal); Writing-review and editing (equal). Christoph Daniel: Resources (equal); Writing-review and editing (equal). Kerstin Amann: Resources (equal); Writing-review and editing (equal). Uwe Zimmermann: Resources (equal); Writingreview and editing (equal). Panagiotis Kavvadas: Investigation (equal); Methodology (equal); Resources (equal); Writing-review and editing (equal). Olaf Grisk: Resources (equal); Writing-review and editing (equal). Christos Chadjichristos: Investigation (equal); Methodology (equal); Resources (equal); Writing-review and editing (equal). Karlhans Endlich: Funding acquisition (equal); Project administration (equal); Supervision (equal); Writing-review and editing (equal). Christos Chatziantoniou: Methodology (equal); Project administration (equal); Supervision (equal). Nicole Endlich: Conceptualization (lead); Funding acquisition (lead); Project administration (lead); Writing-original draft (equal); Writing-review and editing (equal).

\section{DATA AVAILABILITY STATEMENT}

ImageJ macro scripts are available on http://www.github.com/siege rist. Raw microscopy data are available upon request to the authors.

\section{ORCID}

Florian Siegerist (iD https://orcid.org/0000-0003-1629-4982

\section{REFERENCES}

1. Kestilä M, Lenkkeri U, Männikkö M, et al. Positionally cloned gene for a novel glomerular protein-nephrin-is mutated in congenital nephrotic syndrome. Mol Cell. 1998;1:575-582.

2. Reeves W, Caulfield JP, Farquhar MG. Differentiation of epithelial foot processes and filtration slits: sequential appearance of occluding junctions, epithelial polyanion, and slit membranes in developing glomeruli. Lab Invest. 1978;39:90-100.

3. Donoviel DB, Freed DD, Vogel $\mathrm{H}$, et al. Proteinuria and perinatal lethality in mice lacking NEPH1, a novel protein with homology to NEPHRIN. Mol Cell Biol. 2001;21:4829-4836.
4. Boute N, Gribouval O, Roselli S, et al. NPHS2, encoding the glomerular protein podocin, is mutated in autosomal recessive steroidresistant nephrotic syndrome. Nat Genet. 2000;24:349-354.

5. Shih NY, Li J, Karpitskii V, et al. Congenital nephrotic syndrome in mice lacking CD2-associated protein. Science. 1999;286:312-315.

6. Ciani L, Patel A, Allen ND, ffrench-Constant C. Mice lacking the giant protocadherin mFAT1 exhibit renal slit junction abnormalities and a partially penetrant cyclopia and anophthalmia phenotype. Mol Cell Biol. 2003;23:3575-3582.

7. Caulfield JP, Reid JJ, Farquhar MG. Alterations of the glomerular epithelium in acute aminonucleoside nephrosis. Evidence for formation of occluding junctions and epithelial cell detachment. Lab Invest. 1976;34:43-59.

8. Fukasawa H, Bornheimer S, Kudlicka K, Farquhar MG. Slit diaphragms contain tight junction proteins. J Am Soc Nephrol. 2009;20:1491-1503.

9. Furuse M, Tsukita S. Claudins in occluding junctions of humans and flies. Trends Cell Biol. 2006;16:181-188.

10. Tsukita S, Yamazaki Y, Katsuno T, Tamura A. Tight junction-based epithelial microenvironment and cell proliferation. Oncogene. 2008;27:6930-6938.

11. Koda R, Zhao L, Yaoita E, et al. Novel expression of claudin-5 in glomerular podocytes. Cell Tissue Res. 2011;343:637-648.

12. Siegerist F, Ribback S, Dombrowski F, et al. Structured illumination microscopy and automatized image processing as a rapid diagnostic tool for podocyte effacement. Sci. Rep. 2017;7:376.

13. Schordan S, Grisk O, Schordan E, et al. OPN deficiency results in severe glomerulosclerosis in uninephrectomized mice. Am J Physiol Renal Physiol. 2013;304:F1458-F1470.

14. Ransick A, Lindström NO, Liu J, et al. Single-cell profiling reveals sex, lineage, and regional diversity in the mouse kidney. Dev Cell. 2019;51:399-413.e7.

15. Lindenmeyer MT, Eichinger F, Sen K, et al. Systematic analysis of a novel human renal glomerulus-enriched gene expression dataset. PLoS One. 2010;5:e11545.

16. Uhlén M, Fagerberg L, Hallström BM, et al. Proteomics. Tissuebased map of the human proteome. Science. 2015;347:1260419.

17. Nakagawa S, Nishihara K, Miyata H, et al. Molecular markers of tubulointerstitial fibrosis and tubular cell damage in patients with chronic kidney disease. PLoS One. 2015;10:e0136994.

18. Schindelin J, Arganda-Carreras I, Frise E, et al. Fiji: an opensource platform for biological-image analysis. Nat Methods. 2012;9:676-682.

19. Ichimura K, Miyaki T, Kawasaki Y, Kinoshita M, Kakuta S, Sakai T. Morphological processes of foot process effacement in puromycin aminonucleoside nephrosis revealed by FIB/SEM tomography. J Am Soc Nephrol. 2019;30:96-108.

\section{SUPPORTING INFORMATION}

Additional supporting information may be found online in the Supporting Information section.

How to cite this article: Tesch F, Siegerist F, Hay E, et al. Super-resolved local recruitment of CLDN5 to filtration slits implicates a direct relationship with podocyte foot process effacement. J Cell Mol Med. 2021;00:1-11. https://doi. org/10.1111/jcmm.16519 